S67 WNT11 INHIBITS TGFB-INDUCED EPITHELIALMESENCHYMAL TRANSITION IN A 3-DIMENSIONAL HUMAN LUNG TISSUE MODEL

doi:10.1136/thoraxjnl-2012-202678.073

'D Bartis, 'VK D'Souza, ' 0 Wang, ${ }^{2} J E$ Pongracz, 'DR Thickett. 'Department of Clinical Respiratory Sciences, University of Birmingham, Birmingham, United Kingdom, ${ }^{2}$ Department of Medical Biotechnology, University of Pecs, Pecs, Hungary

TGF $\beta 1$ has been implicated as a key effector in mediating Epithelial-Mesenchymal Transition (EMT), my ofibroblastic transdifferentiation and fibroproliferation in the fibrotic diseases of the lung. ATII cells show high differentiational plasticity: either transdifferentiation into ATI or a myofibroblastic phenotype might occur during the disease course. Factors regulating alveolar epithelial differentiation and EMT have high importance in lung regeneration therapy. The support of mesenchymal cells likely contributes to the homeostasis and regeneration of pulmonary epithelium. To study these mesenchymal effects, we constructed a 3-dimensional (3D) human tissue model of primary human ATIIs and pulmonary fibroblasts to mimic epithelial-mesenchymal interactions in the lung.

ATII cells were obtained from lung cancer resection specimens from patients with normal lung function $(n=10)$. ATII cells in the $3 \mathrm{D}$ model retain more differentiated epithelial phenotype compared to conventionally cultured cells $(n=4)$. This is indicated by the higher expression levels of Aquaporin $(A O P) 3(p=0.04)$ and AOP5 $(p=0.19)$, Surfactant Protein (SP)-C $(p=0.11)$ and E-cadherin. $(p=0.37)$ Cells cultured in 2D showed elevated markers of EMT over time whereas 3D cultured cells had significantly lower expression of mesenchymal markers N-cadherin $(p<0.01)$, $\alpha$-Smooth Muscle Actin $(\alpha S M A, p<0.01)$ and the transcription factor SLUG $(p<0.01)$. This suggests that our co-culture model is more relevant in modelling lung diseases in vitro than monolayer epithelial cultures.

We found that externally added recombinant Wnt11 (administered at $1 \mu \mathrm{g} / \mathrm{ml}$ ) inhibits phenotypic changes induced by TGF $\beta 1$ in $3 \mathrm{D}$ ATII+fibroblast co-cultures $(n=6)$. Lung epithelial differentiation markers AQP3 $(p=0.19)$, AOP5 $(p=0.04)$, and SP-C $(p=0.12)$ showed elevated expression levels upon the addition of Wnt11 to TGF $\beta 1$-treated cell cultures. Moreover, $1 \mu \mathrm{g} / \mathrm{ml}$ Wnt11 blocked the EMT-inducing effects of $10 \mathrm{ng} / \mathrm{ml} \mathrm{TGF} \beta 1$ in terms of the expression levels of SLUG $(p=0.01)$, Vimentin $(p<0.01)$ and $\alpha$ SMA $(p=0.04)$.

Our findings indicate that Wnt11 - which is naturally secreted by pulmonary cells - might contribute to the homeostasis and repair of epithelial cells. Our finding that the effects of TGF $\beta 1$ can be antagonised by Wnt11 may mark Wnt11 as a potential therapeutic target in fibrotic diseases of lung.

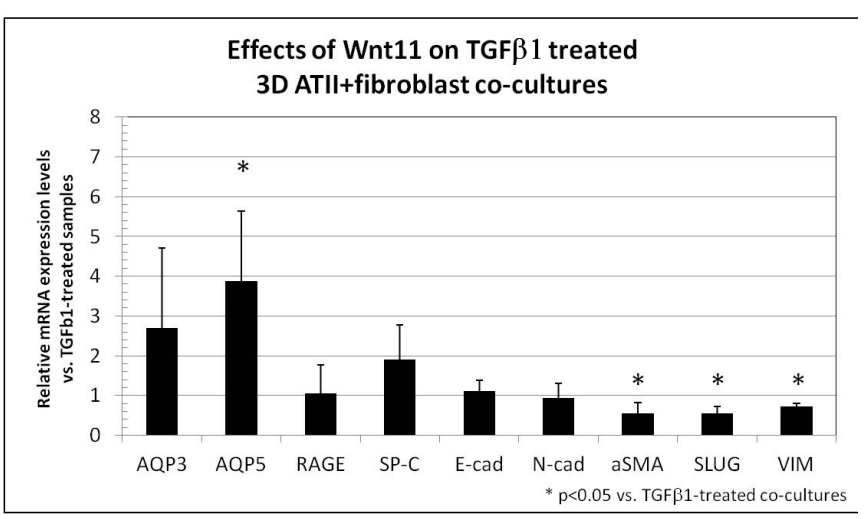

Abstract S67 Figure 1

\section{S68 BIOAVAILABILITY OF VEGF IN IDIOPATHIC PULMONARY FIBROSIS}

doi:10.1136/thoraxjnl-2012-202678.074

${ }^{1}$ SL Barratt, ${ }^{1} \mathrm{C}$ Jarrett, ${ }^{1} \mathrm{~T}$ Blythe, ${ }^{1} \mathrm{GI}$ Welsh, ${ }^{2 \mathrm{~T}}$ Maher, ${ }^{3} \mathrm{D} \mathrm{D}$ Bates, ${ }^{1} \mathrm{AB}$ Millar. ${ }^{1}$ Academic Respiratory Unit, Bristol University, Bristol, UK; ${ }^{2}$ Royal Brompton Hospitals Trust, London, UK: ${ }^{3}$ Microvascular Research Laboratories, Bristol University, Bristol, UK

Introduction Vascular endothelial growth factor (VEGF) is both a growth and permeability factor implicated in the pathogenesis of idiopathic pulmonary fibrosis (IPF). VEGF exerts its biological effects through receptors: VEGFR-1, VEGFR-2, Neuropilin-1(NRP-1) and Neuropilin-2 (NRP-2). The presence of hypoxia and its treatment by high flow oxygen have been proposed to contribute to lung injury. VEGF gene transcription is tightly regulated by a hypoxia response element (HRE).

We hypothesised that:

1. VEGF and its receptors would be differentially expressed between normal (NF) and fibrotic fibroblasts (FF).

2. Hypoxia and hyperoxia may alter fibroblast VEGF expression.

Methods $\mathrm{NF}(\mathrm{n}=5)$ and $\mathrm{FF}(\mathrm{n}=5)$ (from patients with proven usual interstitial pneumonia) were extracted from lung biopsies using the explant method. VEGF receptors levels were analysed at the mRNA and protein level (qPCR and western blotting (WB)). Pan VEGF isoforms were detected at the protein level using WB and ELISA. Fibroblasts were grown in culture for 24 hours in normoxic, hyperoxic $\left(90 \% \mathrm{O}_{2}\right)$ and hypoxic conditions $\left(21 \% \mathrm{O}_{2}\right.$ with $\left.\mathrm{CoCl}_{2}\right)$.

Results Both NF and FF expressed VEGFR1, VEGFR2, NRP-1 and NRP-2. No significant difference was detected in receptor expression at mRNA or protein level, but a trend towards reduced protein expression of VEGFR1 and NRP-2 in FF vs NF was observed. FF expressed significantly more total VEGF than NF by ELISA (Figure 1): (NF $180.5 \mathrm{pg} / \mathrm{ml}$ vs FF $332.0 \mathrm{pg} / \mathrm{ml}, \mathrm{p}=0.01$ ) and by WB (normalised densitometry value: NF 116 vs 162, $\mathrm{p}=0.046$ ). Moreover, a significant increase in VEGF expression was observed in both normal and fibrotic fibroblasts in response to hypoxic growth conditions (NF $205.7 \mathrm{pg} / \mathrm{ml}$ vs $1382.0 \mathrm{pg} / \mathrm{ml}, \mathrm{p}=0.05$, FF $394.6 \mathrm{pg} / \mathrm{ml}$ vs $1113 \mathrm{pg} /$ $\mathrm{ml} .0, \mathrm{p}=0.01)$. A trend towards increased VEGF expression was also seen in FFvsNF exposed to hyperoxic conditions.

Conclusions Differential expression of VEGF between NF and FF suggests a potential role in the development of IPF. The observation that hypoxia and possibly hyperoxia may alter VEGF bioavailability has implications in the use of oxygen therapy in the management of this disease.

\section{S69 TRANSCRIPTIONAL MECHANISMS REGULATING EXPRESSION OF THE AVBG INTEGRIN IN IPF}

doi:10.1136/thoraxjn-2012-202678.075

${ }^{1}$ AL Tatler, 'G Saini, 'A Goodwin, '10 Gbolohan, 'RL Clifford, 'M Al'Hourani, 'J Porte, ${ }^{2} \mathrm{~S}$ Violette, ${ }^{2} \mathrm{P}$ Weinreb, ${ }^{1} \mathrm{~A}$ Knox, ${ }^{3} \mathrm{G}$ Laurent, ${ }^{4} \mathrm{P}$ Wolters, ${ }^{5} \mathrm{~J}$ Gauldie, ${ }^{2} \mathrm{M}$ Kolb, ${ }^{1} \mathrm{G}$ Jenkins 'University of Nottingham, Nottingham, UK; ${ }^{2}$ Biogen Idec, Cambridge, USA; ${ }^{3}$ University College London, London, UK; ' University of California San Francisco, San Francisco, USA; ${ }^{5}$ McMaster University, Hamilton, Canada

TGF- $\beta$ is fibrogenic cytokine implicated in the pathogenesis of idiopathic pulmonary fibrosis (IPF). The integrin $\alpha \mathrm{V} \beta 6$ can activate TGF- $\beta$, and dysregulation of this pathway is thought to play a role in the pathogenesis of pulmonary fibrosis. TGF- $\beta$ induces $\alpha \mathrm{V} \beta 6$ integrin expression and aVb6 integrins are upregulated in fibrotic regions of lungs from patients with IPF. This raises the possibility that dysregulation of a self-regulating feedback loop may promote IPF. This study aims to investigate the mechanisms involved TGF$\beta$-induced $\beta 6$ expression and how this may be dysregulated in IPF.

Using QPCR and flow cytometry we assessed expression of the integrin $\beta 6$ (ITGB6) in lung epithelial cells. An ITGB6 promoterluciferase construct and truncated mutants were used to identify 